\title{
DNA Resequencing
}

National Cancer Institute

\section{Source}

National Cancer Institute. DNA Resequencing. NCI Thesaurus. Code C41254.

A specific application of gene sequencing that precisely determines the sequence of bases in DNA. Resequencing is used to identify single nucleotide polymorphisms (SNPs). 